\title{
Epidermal growth factor or platelet-rich plasma combined with induced membrane technique in the treatment of segmental femur defects: an experimental study
}

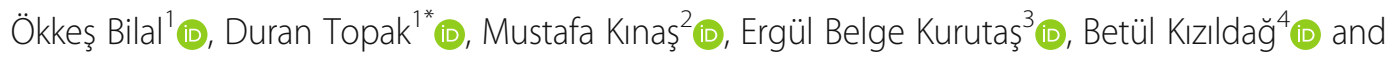
Abdulkadir Yasir $\operatorname{Bahar}^{5}$ (D)

\begin{abstract}
Objective: Extensive bone defects remain a therapeutic challenge necessitating alternative surgical approaches with better outcomes. Can increase the effectiveness of PRP or EGF treatment in surgical treatment of large bone defects with Masquelet technique?

Aim of this study examined potential therapeutic benefits of the Masquelet technique with induced membranes in combination with platelet-rich plasma (PRP) or epidermal growth factor (EGF) in a rat model of segmental femur defect.
\end{abstract}

Methods: Three groups each consisting of 20 Sprague-Dawley rats were defined as follows: EGF group, PRP group, and control group. A femoral bone defect was created and filled with antibiotic embedded polymethyl methacrylate. Half of the animals in each group were sacrificed at week 6 and the pseudo-membranes formed were analyzed. In the remaining half, the cement was removed and the space was filled with autograft. After another 6 weeks, the structures formed were examined radiologically, histologically, and biochemically.

Results: At week 6, both PRP and EGF groups had significantly higher membrane CD31, TGF-beta, and VEGF levels than controls. At week 12, when compared to controls, PRP and EGF groups had significantly higher membrane CD31 levels and the PRP group had significantly higher membrane TGF levels. Regarding bone tissue levels, PRP and EGF groups had significantly higher VEGF levels and the EGF group had significantly higher BMP levels. In addition, PRP and EGF groups had higher radiological scores than controls. However, the two experimental groups did not differ with respect to any parameter tested in this study.

Conclusion: Both PRP and EGF seem to be associated with histological, biochemical, and radiological improvements in experimental rat model of Masquelet technique, warranting in further clinical studies.

Level of evidence: Level 5

Keywords: Epidermal growth factor (EGF), Platelet-rich plasma (PRP), Masquelet technique, Induced membrane, Experimental study, Femur

\footnotetext{
* Correspondence: drdtopak@gmail.com

'Department of Orthopaedic and Traumatology, Faculty of Medicine,

Kahramanmaras Sutcu Imam University, Kahramanmaras, Turkey

Full list of author information is available at the end of the article
}

\section{$\triangle B M C$}

(c) The Author(s). 2020 Open Access This article is licensed under a Creative Commons Attribution 4.0 International License, which permits use, sharing, adaptation, distribution and reproduction in any medium or format, as long as you give appropriate credit to the original author(s) and the source, provide a link to the Creative Commons licence, and indicate if changes were made. The images or other third party material in this article are included in the article's Creative Commons licence, unless indicated otherwise in a credit line to the material. If material is not included in the article's Creative Commons licence and your intended use is not permitted by statutory regulation or exceeds the permitted use, you will need to obtain permission directly from the copyright holder. To view a copy of this licence, visit http://creativecommons.org/licenses/by/4.0/ The Creative Commons Public Domain Dedication waiver (http://creativecommons.org/publicdomain/zero/1.0/) applies to the data made available in this article, unless otherwise stated in a credit line to the data. 


\section{Introduction}

Extensive bone defects due to traumatic injury remain a therapeutic challenge in terms of anatomical and functional outcomes. The traditional approach for restoration of bone defects requires extensive surgical intervention involving the use of bone grafting that is associated with donor-site complications and frequent occurrence of morbidity, with no guarantee of complete correction of the defect [1]. Thus, the search for alternative surgical approaches continues. Recently, Masquelet technique has been described as a two-stage treatment strategy for large bone defects that consists of a temporary cement spacer followed by bone grafting [2]. The technique allows reconstruction of extensive diaphysis defects even in the presence of previous radiation exposure or infections.

Platelet-rich plasma (PRP) is a volume of fractionated plasma from patient's own blood containing platelet concentrate [3]. PRP contains several growth factors that play a major role in tissue repair mechanisms including but not limited to platelet-derived growth factor, transforming growth factor-beta, and vascular endothelial growth factor. As a result of its potential therapeutic effects, PRP has recently gained significant attention as a safe nonsurgical adjunctive treatment modality for osteoarthritis and musculoskeletal repair [4]. Despite the lack of definitive evidence for the therapeutic benefit of PRP in bone healing $[5,6]$, a multitude of recent experimental and clinical publications have suggested a potential utility [7-13].

As compared to PRP, however, published data on the use of epidermal growth factor (EGF) in orthopedic surgery is very scarce and most information relates to its potential therapeutic benefits in conditions other than bone healing such as wound healing, diabetic foot ulcers, or experimental dentistry [14-18].

In the meta-analysis study of the Masquelet technique performed by Morelli et al., it was shown that bone union occurred in $89.7 \%$ of the cases, and the rest did not [19].

This study was undertaken as an initial experimental attempt to assess the feasibility and potential therapeutic benefits of the Masquelet technique with induced membranes in combination with PRP and EGF in a rat model of segmental femur defect. The assessments included comparison of tissue bone morphogenetic protein 2 (BPM-2), transforming growth factor-beta (TGF-beta), and vascular endothelial growth factor (VEGF) levels as markers of bone healing, osteoinduction, and angiogenesis, as well as histopathological and radiological examinations in experimental study groups.

\section{Materials and methods}

\section{Design and experimental animals}

A total of 60 male Sprague-Dawley rats weighing between 400 and $450 \mathrm{~g}$ were used for the experimental protocol. Three groups each consisting of 20 rats were defined as follows: EGF group, PRP group, and control group. Standard housing and feeding conditions were provided for the animals. The study protocol was approved by Kahramanmaras Sutcu Imam University Medical Faculty Animal Experimentation Ethics Committee (session: 2014/01, date: 17.04.2014, decision no: 05).

A 5-mm bone defect was created and filled with antibiotic embedded polymethyl methacrylate followed by the fixation of the femur using external fixators. Figure 1 shows the defect model. Half of the animals in each group were sacrificed at week 6 and the pseudomembranes formed were prepared for study analyses. In the remaining half of the groups, the cement was removed and the resultant space was filled with autograft obtained from the tail of each animal. After 6 weeks, the bony structures formed were examined radiologically, in addition to histological and biochemical assessments.

\section{Procedures (first step)}

Prior to surgery, each animal received a single $0.1 \mathrm{mg} / \mathrm{kg}$ intramuscular dose of cefazolin sodium (Cefozin, Bilim Ilac, Turkey) prophylactically. For surgery, anesthesia was provided with intramuscular ketamine $\mathrm{HCl} 200 \mathrm{mg} /$ $\mathrm{kg}$ (Ketalar Eczacibasi, Turkey) and xylazine $1 \mathrm{mg} / \mathrm{kg}$ (RompunR, Bayer, Turkey). Right hind legs were shaved and covered with sterile covers after disinfection with Betadine (polyvidone iodine solution). The skin and subcutaneous tissues were dissected in accordance with surgical principles to expose the femur of rats. A $5-\mathrm{mm}$ bony defect was created using a $1-\mathrm{mm}$ drill tip and osteotome, and then it was filled with antibiotic embedded polymethyl methacrylate (Cemex Genta ID Green TECRES ${ }^{\odot}$ Italy). Femur was fixated using external fixators, and the skin was sutured using 4/Ethilon nylon monofilament suture material. EGF and PRP were injected into the wound area.

Rats in the epidermal growth factor group received, epidermal growth factor was injected into bone defect area at a dose of $25 \mu \mathrm{g} / \mathrm{ml}$ once a week for 3 weeks (HEBERPROT-P ${ }^{\circ} 75 \mu \mathrm{g}$, Hasbiotech Ilac San. ve Tic. A.S. Turkey). The blood obtained from the rats in the PRP group was used to prepare platelet-rich plasma using a MAGELLAN ${ }^{\circledR}$ Autologous Platelet Separator System (Arteriocyte Medical Systems USA). It was injected into the bone defect area once a week for 3 weeks volumes of $1 \mathrm{ml}$. Controls received weekly injections of 1 $\mathrm{ml}$ of physiological saline around the cement. Half of the rats were sacrificed at the end of 6 weeks using high dose pentobarbital anesthesia and the pseudomembranes forming around the space filled with cement were extracted. Each membrane sample was divided into two halves, one being fixed in formaldehyde for histopathological assessments, and the other kept at $-40{ }^{\circ} \mathrm{C}$ 


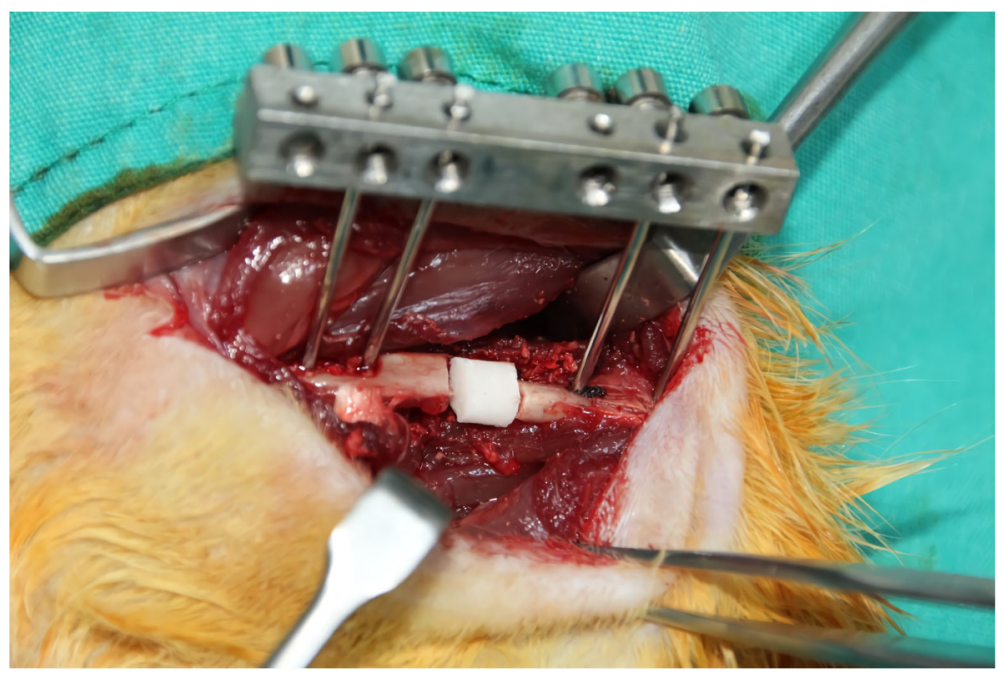

Fig. 1 Segment creation and spacer application with antibiotic-embedded cement in rat femur (blue arrow)

for biochemical analyses. Three animals in the EGF and one in the control group died and were therefore excluded from the analyses.

\section{Procedures (second step)}

In the remaining rats in study groups, the same procedures as described in step one were used to expose the femurs. The pseudo-membranes formed were carefully and longitudinally opened and autogenic bone grafts retrieved from the tail of each animal were placed into the resulting space. The operation site was sutured.

Six weeks after these procedures, anteroposterior and lateral X-rays and CT images were obtained for radiological assessments. The rats were sacrificed with high dose pentobarbital anesthesia, and the pseudomembranes around the defect into which grafts were placed and bone tissue were removed and cut into two equal pieces, one being fixed in formaldehyde for histopathological assessments, and the other kept at $-40{ }^{\circ} \mathrm{C}$ for biochemical analyses. At this stage, 3, 4, and 2 animals died in PRP, EGF, and control groups, respectively, and were therefore excluded from the analyses. Paracetamol was administered to rats in appropriate doses as a pain reliever during the study.

\section{Biochemical assessments}

Rat tissue samples were placed into ice as soon as they were removed, blotted, and weighed. Homogenization for 15 to $20 \mathrm{~min}$ was performed in a homogenizer with ice boxes containing $1 \mathrm{~g}$ of tissue per 5 volume of cold $1.15 \% \mathrm{KCl}$ (weight/volume). Then, the homogenate was centrifuged for $30 \mathrm{~min}$ at $14000 \mathrm{rpm}$ at $+4{ }^{\circ} \mathrm{C}$, and the biochemical analyses were done on the supernatant. ELISA double-sandwich methodology was used for BMP-2 (pg/mL), TGF-beta $(\mathrm{ng} / \mathrm{mL})$, and VEGF $(\mathrm{pg} / \mathrm{mL})$ assessments and BMP-2, VEGF and TGF-beta levels in the tissue samples were measured in duplicate using commercially available solid-phase sandwich enzymelinked immunosorbent assay (ELISA) kits (MyBioSource Company, USA) according to the manufacturer's protocol.

\section{Histopathological assessments}

The samples were fixed for $24 \mathrm{~h}$ in $10 \%$ buffered formaldehyde and then were embedded. After obtaining appropriate cross-sections from paraffin blocks, they were stained with hematoxylin and eosin (HE) and were examined under a light microscope (Olympus-BX53). In order to perform a histochemical assessment of the neovascularization of the pseudo-membrane, granulation tissue was identified in HE stained cross sections. A histopathologist, unaware of the study procedures, was included in the study. Based on its high specificity and sensitivity as an endothelial marker, CD31 (platelet endothelial cell adhesion molecule-PECAM1) was chosen as a marker of endothelial proliferation and neovascularization, and was applied immune histochemically to the areas of granulation identified with HE staining. Cross-sections of 3-micron thickness were stained with CD31 using a "LEICA BENCHMARK XT" immunohistochemistry device and were assessed using a light microscope with quantification of vascular structures (capillaries as well as immature vascular structures) in each $1 \mathrm{~mm}^{2}$ at $\times 40$ magnification power (Fig. 2).

\section{Radiological assessments}

In each rat, antero-posterior and lateral X-rays as well as computed tomography images (Toshiba Alexon 16 multi-slice) were obtained (Fig. 3). A radiologist blinded to the study procedures used a CT version of Lane- 

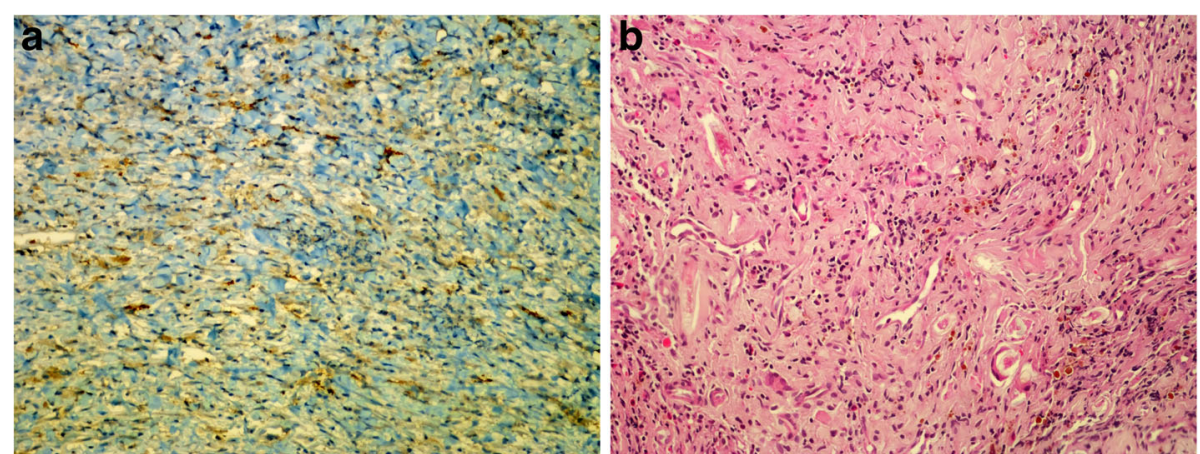

Fig. 2 Pathological images of fracture healing in experimental cases. a Microscopic image of prepatellar stained with CD 31 at $\times 200$ magnification (brown areas indicate endothelial cells stained with CD 31). b Microscopic image of prepatellar stained with hematoxylin eosin at $\times 200$ magnification (areas of inflammatory granulation tissue was shown)

Sandhu scoring system for image scoring as follows: 0 , no callus, clear fracture line; $1,25 \%$ callus tissue, the fracture line can be clearly observed; $2,50 \%$ callus tissue, blurred fracture line; $3,75 \%$ callus tissue, fracture line barely visible; $4,100 \%$ callus tissue, fracture line cannot be seen [19].

\section{Statistical analyses}

Statistical Package for Social Sciences (SPSS) version 21 was used for the analysis of data. Normality was tested using Shaphiro-Wilk test and graphical methods. Kruskal-Wallis test or analysis of variance (ANOVA) was used to test intergroup differences, and built-in post hoc test for Kruskal-Wallis or Tukey HSD was used for pairwise comparisons, respectively. A $p$ value smaller than 0.05 was considered the indication for statistical significance.

\section{Results}

Table 1 shows the comparison of the groups with respect to study assessments, at week 6 and week 12 .

\section{Membrane tissue assessments at week 6}

At week 6, the PRP group had significantly higher membrane CD31 $(p=0.002)$, TGF $(p<0.001)$, and VEGF $(p$ $<0.001)$ levels than controls. Similarly, the EGF group had significantly higher membrane CD31, TGF-beta, and VEGF levels than controls $(p<0.001$ for all three comparisons). However, the two groups did not differ with regard to any of these three parameters.

Biochemical, histological, and radiological assessments at week 12

At week 12, PRP $(p<0.014)$ and EGF $(p<0.008)$ groups had significantly higher membrane CD31 levels than
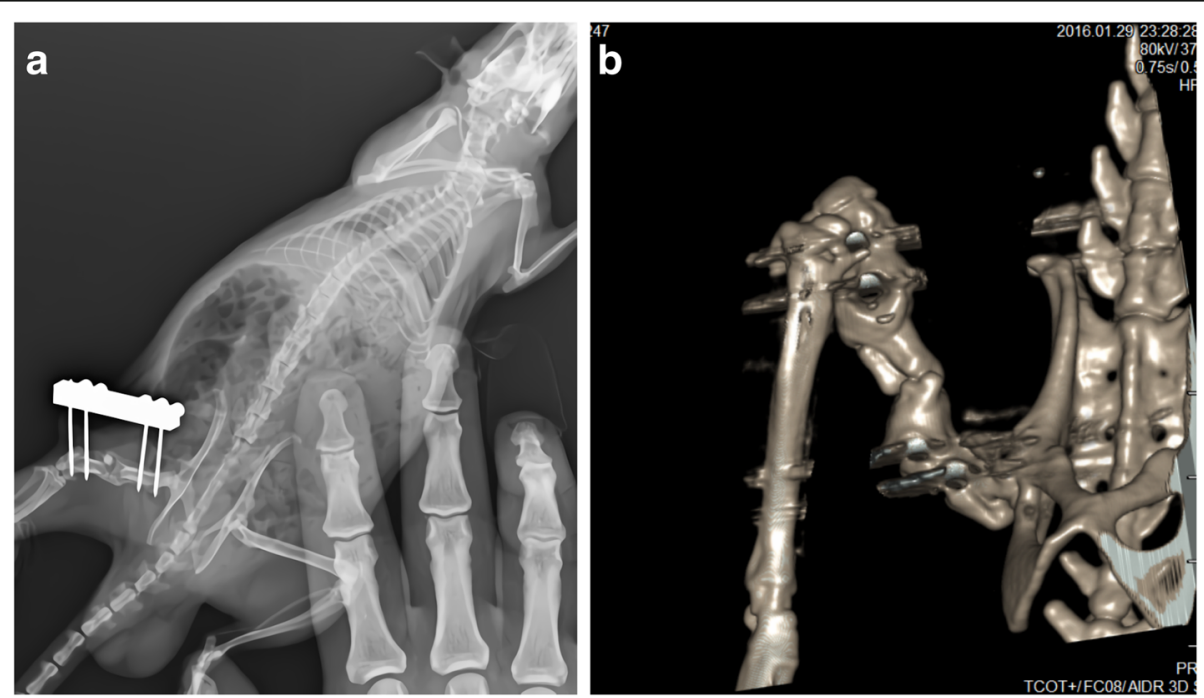

Fig. 3 Radiological image of experimental cases. a Radiographic image of the case in the PRP group after 6 weeks (Lane-Sandhu score 4). b Computed tomography image of the case in the PRP group after 6 weeks (Lane-Sandhu score 4) 
Table 1 Comparison of the groups with regard to histological, biochemical, and radiological study assessments

\begin{tabular}{|c|c|c|c|c|}
\hline & Group 1 (PRP) & Group 2 (EGF) & Group 3 (controls) & $p$ \\
\hline \multicolumn{5}{|l|}{ Assessments at week 6 (mean $\pm \mathrm{SD}$ ) } \\
\hline Histological CD31 assessment (counts/mm²) & $143.7 \pm 8.2$ & $151.1 \pm 9.2$ & $125.2 \pm 13.3$ & $<0.001$ \\
\hline Membrane TGF level (ng/mL) & $246.4 \pm 54.3$ & $257.7 \pm 55.2$ & $141.8 \pm 20.8$ & $<0.001$ \\
\hline Membrane VEGF level (pg/mL) & $842.0 \pm 98.5$ & $754.8 \pm 118.7$ & $430.5 \pm 92.2$ & $<0.001$ \\
\hline \multicolumn{5}{|l|}{ Assessments at week 12 (mean $\pm \mathrm{SD}$ ) } \\
\hline Histological CD31 assessment (counts/mm²) & $137.3 \pm 17.3$ & $138.1 \pm 14.0$ & $99.6 \pm 25.5$ & 0.003 \\
\hline Membrane TGF level (ng/mL) & $397.4 \pm 44.4$ & $379.3 \pm 63.3$ & $326.4 \pm 42.0$ & 0.032 \\
\hline Membrane VEGF level (pg/mL) & $318.0 \pm 95.2$ & $273.0 \pm 72.1$ & $228.9 \pm 44.9$ & 0.086 \\
\hline Bone VEGF level (pg/mL) & $192.2 \pm 6.7$ & $198.1 \pm 5.7$ & $176.3 \pm 7.2$ & 0.003 \\
\hline Bone BMP-2 level (pg/mL) & $191.9 \pm 8.4$ & $237.6 \pm 37.3$ & $117.1 \pm 43.0$ & 0.012 \\
\hline \multicolumn{5}{|l|}{ Lane score (median, range) } \\
\hline Anteroposterior & $4(3-4)$ & $3(2-4)$ & $1(1-2)$ & 0.015 \\
\hline Lateral & $4(2-4)$ & $3.5(2-4)$ & $1(1-2)$ & 0.026 \\
\hline $\mathrm{CT}$ & $3(2-4)$ & $3(2-3)$ & $1(1-2)$ & 0.026 \\
\hline $3 D$ & $3.5(2-4)$ & $4(3-4)$ & $1.5(1-3)$ & 0.044 \\
\hline
\end{tabular}

Abbreviations: PRP platelet rich plasma, EGF epidermal growth factor, CD 31 cluster of differentiation 31, TGF-beta transforming growth factor-beta, BPM-2 bone morphogenetic protein 2, VEGF vascular endothelial growth factor, $C T$ computed tomography, $3 D$ three-dimensional

controls, with no significant difference between the former two groups. PRP group had significantly higher membrane TGF-beta levels than controls $(p=0.032)$; however, no other pairwise difference reached significance.

Regarding bone tissue levels, PRP $(p<0.019)$ and EGF $(p<0.003)$ groups had significantly higher VEGF levels than controls, without any significant difference between the former two groups. On the other hand, the EGF group had significantly higher bone BMP levels than controls $(p=0.010)$, with no other significant pairwise differences.

\section{Lane scores at week 12}

PRP group had significantly higher AP $(p=0.013)$, lateral $(p=0.040)$, and CT scores $(p=0.040)$ than controls, whereas no other pairwise difference was evident regarding these parameters. On the other hand, the EGF group had significantly higher $3 \mathrm{D}$ scores than controls $(p=$ 0.047 , representing the only significant pairwise difference for this parameter.

\section{Discussion}

This study examining the effect of two therapeutic approaches, i.e., PRP and EGF, on bone healing when used as an adjunct to the Masquelet technique for the management extensive bone defects has provided promising results in terms of a potential therapeutic benefit. Accordingly, both PRP and EGF were associated with improved biochemical and histological outcomes, both at 6- and 12-week assessment timepoints, as reflected by higher membrane/bone tissue CD31, TGF-beta, and
VEGF levels in PRP and EGF groups. In addition, these adjunctive treatments to the Masquelet technique resulted in radiological improvements in AP lateral X-ray and CT scores (for PRP) and 3D scores (EGF). Of these two approaches, PRP has previously been subject to extensive research regarding its therapeutic utility in orthopedic procedures, while literature data on EGF is rather scarce. Furthermore, to the best of our knowledge, no previous studies have examined these two agents in combination with the Masquelet technique in such an experimental setting.

PRP has been generally reviewed as "a promising therapeutic approach for future regenerative treatments" [4] and a great majority of the preclinical studies on the treatment of bone defects support the use of PRP [5]. Specifically, positive results have been reported in rabbit ulnar defects [20], rat femoral fractures [11], as well as in rabbit tibia shaft fractures [21]. In humans, clinical reports on the healing rate of long bone non-union fractures have also indicated positive outcomes in terms of cure rate, healing duration, and limb shortening $[8,9]$. Despite these initial promising results, it has been underscored that the evidence to support the routine use of this intervention in clinical practice is currently insufficient [10].

In contrast with PRP, research on the possible therapeutic effects of epidermal growth factor (EGF) in tissue healing is mainly limited to the field of experimental dentistry [15, 17], chronic diabetic foot ulcers [14], or other types of soft tissue pathologies including chronic radiation ulcers [18]. On the other hand, EGF receptors are known to play a role in endochondral bone 
formation [22], justifying research assessing its potential effects in bone healing. For instance, loss of epidermal growth factor receptor (EGFR) activity was found to alter the development of the growth plate, impair endochondrial ossification, and retard the growth in a mice model [22]. Furthermore, administration of EGF in liposomes resulted in a faster recovery of injured alveoli of rats after the extraction of the maxillary second molar teeth, providing protective effects against early absorption and degradation [17]. In a case report exploring the potential benefits of recombinant epidermal growth factor in a patient with radiation-induced chronic wound, successful healing within 16 weeks was reported after the failure of flap surgery and conventional treatments given over a $3=y e a r$ period [18]. Similar enhancement of wound healing leading to thicker epidermal and dermal layers was also reported with the use of decellularized scaffolds loaded with epidermal growth factor in an experimental model of wound healing [16]. Overall, our results are in concordance with the abovementioned findings as reflected by improved biochemical, histopathological, as well as radiological outcomes in rats receiving additional EGF treatment as a part of the Masquelet technique for segmental bone defects.

Production of growth factors (VEGF, TGF-beta) as well as osteoinductive factors (BMP-2) has been previously documented in the induced membranes in a rabbit model of the Masquelet technique [23]. Furthermore, platelets are known to contain alpha-granules that are rich in a number of growth factors including plateletderived growth factor, transforming growth factor-beta, insulin-like growth factor, vascular endothelial growth factor, and epidermal growth factor, which are of major significance in tissue repair mechanisms [4].

Thus, our results corroborate previous studies and provide further evidence for the first time that PRP and EGF have the potential to be used as an adjunct for accelerated healing in the Masquelet technique for the management of extensive bone defects. However, the small sample size is certainly a limitation of our study. Further studies may confirm our findings for each of these agents and may possibly explore the utility of the combined use of these two agents for potential synergistic therapeutic effects.

A limitation of the current study could be considered to be the low sample size in the groups, but the number was appropriate according to the power analysis calculation. Although CD31 was found to be sufficient for vascularization analysis in rats, other vascularization indicators such as vWF could be added.

\section{Conclusions}

Masquelet technique is an effective surgical method that can be applied easily in the restoration of large bone defects. In the Masquelet technique, pseudomembrane formation is the main factor in the restoration of bone defect. The addition of EGF or PRP to the Masquelet technique has been shown to increase vascularization in the pseudomembrane.

Histopathological and biochemical healing displayed by the addition of EGF or PRP to the Masquelet technique in the restoration of large bone defects also provided a radiologically significant improvement.

Both PRP and EGF were associated with improved histological, biochemical, and radiological improvements in this experimental rat model of the Masquelet technique. These promising observations may be confirmed in further clinical studies involving humans.

\section{Acknowledgements}

Not applicable.

\section{Animal and human rights statement}

All procedures performed in this study were in accordance with the ethical standards of the institutional and/or national research committee and with the 1964 Helsinki Declaration and its later amendments or comparable ethical standards. No animal or human studies were carried out by the authors for this article.

None of the authors received any type of financial support that could be considered potential conflict of interest regarding the manuscript or its submission.

\section{Authors' contributions}

Ökkeş Bilal, Duran Topak, and Mustafa Kinaş constructed the idea or hypothesis for research; Ökkeş Bilal and Duran Topak took the responsibility in drafting of the whole the manuscript; Ökkeş Bilal and Betül Kizildağ reviewed the article before submission not only for spelling and grammar but also for its intellectual content; Ergül Belge Kurutaş and Abdulkadir Yasir Bahar took responsibility in drafting of the project and ethic paper; Ökkeş Bilal, Duran Topak, and Betül Kizildağ took the responsibility in the execution of the experiments, data management, and reporting. All authors reviewed and approved the final manuscript.

\section{Funding}

This study was supported by the research projects management department of Kahramanmaraş Sütçü Imam University (2015/1-60M).

\section{Availability of data and materials}

The data and materials of patients participating in this study are available to us and will be provided by us upon request.

\section{Ethics approval and consent to participate}

The study protocol was approved by the Local Ethics Committee for Animal Experimentation, Kahramanmaras Sutcu Imam University with an approval date and no. of 2014/01/05 and 17 April 2014, respectively.

Consent for publication

All authors have agreed to publish this content in your journal.

\section{Competing interests}

The authors declare that there is no conflict of interest.

\section{Author details}

${ }^{1}$ Department of Orthopaedic and Traumatology, Faculty of Medicine, Kahramanmaras Sutcu Imam University, Kahramanmaras, Turkey. ${ }^{2}$ Private Bandırma Royal Hospital, Balıkesir, Turkey. ${ }^{3}$ Department of Biochemistry, Faculty of Medicine, Kahramanmaras Sutcu Imam University, Kahramanmaras, Turkey. ${ }^{4}$ Department of Radiology, Faculty of Medicine, Kahramanmaras Sutcu Imam University, Kahramanmaras, Turkey. ${ }^{5}$ Department of Pathology, Faculty of Medicine, Kahramanmaras Sutcu Imam University, Kahramanmaras, Turkey. 
Received: 23 September 2020 Accepted: 30 November 2020

Published online: 11 December 2020

\section{References}

1. Hertel R, Gerber A, Schlegel U, Cordey J, Ruegsegger P, Rahn BA. Cancellous bone graft for skeletal reconstruction. Muscular versus periosteal bed-preliminary report. Injury. 1994;25(Suppl 1):A59-70.

2. Masquelet AC, Begue T. The concept of induced membrane for reconstruction of long bone defects. Orthop Clin North Am. 2010;41(1):2737 table of contents

3. Marx RE. Platelet-rich plasma (PRP): what is PRP and what is not PRP? Implant Dent. 2001;10(4):225-8.

4. Dhillon RS, Schwarz EM, Maloney MD. Platelet-rich plasma therapy - future or trend? Arthritis Res Ther. 2012;14(4):219.

5. Roffi A, Di Matteo B, Krishnakumar GS, Kon E, Filardo G. Platelet-rich plasma for the treatment of bone defects: from pre-clinical rational to evidence in the clinical practice. A systematic review. Int Orthop. 2017:41(2):221-37.

6. Sheth $U$, Simunovic N, Klein G, et al. Efficacy of autologous platelet-rich plasma use for orthopaedic indications: a meta-analysis. J Bone Joint Surg Am. 2012:94(4):298-307.

7. Gawai KT, Sobhana CR. Clinical evaluation of use of platelet rich plasma in bone healing. J Maxillofac Oral Surg. 2015;14(1):67-80.

8. Ghaffarpasand F, Shahrezaei M, Dehghankhalili M. Effects of platelet rich plasma on healing rate of long bone non-union fractures: a randomized double-blind placebo controlled clinical trial. Bull Emerg Trauma. 2016;4(3): 134-40

9. Golos J, Walinski T, Piekarczyk P, Kwiatkowski K. Results of the use of platelet rich plasma in the treatment of delayed union of long bones. Ortop Traumatol Rehabil. 2014;16(4):397-406.

10. Griffin XL, Wallace D, Parsons N, Costa ML. Platelet rich therapies for long bone healing in adults. Cochrane Database Syst Rev. 2012;7:CD009496.

11. Guzel Y, Karalezli N, Bilge O, et al. The biomechanical and histological effects of platelet-rich plasma on fracture healing. Knee Surg Sports Traumatol Arthrosc. 2015:23(5):1378-83.

12. Malhotra R, Kumar V, Garg B, et al. Role of autologous platelet-rich plasma in treatment of long-bone nonunions: a prospective study. Musculoskelet Surg. 2015;99(3):243-8

13. Zhang ZY, Huang AW, Fan JJ, et al. The potential use of allogeneic plateletrich plasma for large bone defect treatment: immunogenicity and defect healing efficacy. Cell Transplant. 2013;22(1):175-87.

14. Fernandez-Montequin Jl, Betancourt BY, Leyva-Gonzalez G, et al. Intralesional administration of epidermal growth factor-based formulation (Heberprot-P) in chronic diabetic foot ulcer: treatment up to complete wound closure. Int Wound J. 2009;6(1):67-72.

15. Furfaro F, Ang ES, Lareu RR, Murray K, Goonewardene M. A histological and micro-CT investigation in to the effect of NGF and EGF on the periodontal, alveolar bone, root and pulpal healing of replanted molars in a rat model a pilot study. Prog Orthod. 2014;15:2.

16. Su Z, Ma H, Wu Z, et al. Enhancement of skin wound healing with decellularized scaffolds loaded with hyaluronic acid and epidermal growth factor. Mater Sci Eng C Mater Biol Appl. 2014:44:440-8.

17. Marquez L, de Abreu FA, Ferreira CL, Alves GD, Miziara MN, Alves JB Enhanced bone healing of rat tooth sockets after administration of epidermal growth factor (EGF) carried by liposome. Injury. 2013;44(4):558-64.

18. Lee SW, Moon SY, Kim YH, Hong JP. The use of recombinant human epidermal growth factor to promote healing for chronic radiation ulcer. Int Wound J. 2007:4(3):216-20

19. Lane JM, Sandhu HS. Current approaches to experimental bone grafting. Orthop Clin North Am. 1987;18(2):213-25.

20. Galanis V, Fiska A, Kapetanakis S, Kazakos K, Demetriou T. Effect of plateletrich plasma combined with demineralised bone matrix on bone healing in rabbit ulnar defects. Singapore Med J. 2017;58(9):551-6.

21. Kanthan SR, Kavitha G, Addi S, Choon DS, Kamarul T. Platelet-rich plasma (PRP) enhances bone healing in non-united critical-sized defects: a preliminary study involving rabbit models. Injury. 2011;42(8):782-9.
22. Zhang $X$, Siclari VA, Lan $S$, et al. The critical role of the epidermal growth factor receptor in endochondral ossification. J Bone Miner Res. 2011;26(11): 2622-33.

23. Pelissier P, Masquelet AC, Bareille R, Pelissier SM, Amedee J. Induced membranes secrete growth factors including vascular and osteoinductive factors and could stimulate bone regeneration. J Orthop Res. 2004;22(1):73-9.

\section{Publisher's Note}

Springer Nature remains neutral with regard to jurisdictional claims in published maps and institutional affiliations.
Ready to submit your research? Choose BMC and benefit from:

- fast, convenient online submission

- thorough peer review by experienced researchers in your field

- rapid publication on acceptance

- support for research data, including large and complex data types

- gold Open Access which fosters wider collaboration and increased citations

- maximum visibility for your research: over $100 \mathrm{M}$ website views per year

At $\mathrm{BMC}$, research is always in progress.

Learn more biomedcentral.com/submissions 